\title{
Diffusion en mer des rejets des systèmes d'assainissement et gestion opérationnelle de leurs impacts sur la qualité des eaux de baignade
}

\author{
Sanitation systems discharge diffusion into sea and operational \\ management of their impacts on bathing water quality \\ EMMANUEL SOYEUX \\ Veolia Environnement \\ Direction de la Recherche du Développement et de la Technologie - 36-38 avenue Kléber - 75799 Paris Cedex 16 \\ Tél: +33 (0)1 01717508 58, Fax: +33 (0)101717505 93, e-mail: emmanuel.soyeux@veolia.com
}

YANN-HERVE DE ROECK

Ifremer

Département Dynamiques de l'Environnement Côtier - BP 70 - F-29 280 Plouzané

Tél: +33 (0)2 982244 95, Fax: +33 (0)2 982208 58, e-mail: yhdr@ifremer.fr

\begin{abstract}
$S$ anitation systems discharge diffusion into sea and operational management of their impacts on bathing water quality The new European Directive concerning the management of bathing water quality drives the littoral communities towards operational management tools for an actual management of the beaches according to the impacts of the urban discharges. Beyond the progressive modernisation of the sanitation system itself, taking into account globally all the chronic factors impacting the beaches is only made possible by using decisionmaking systems based on a better knowledge of the sources of contamination and on the numerical modelling of the diffusion into the sea. Such a system, easy to operate, was developed by Veolia and Ifremer for the agglomeration of Dieppe (Normandy, France): it queries a database of pre-computed scenarios of conditions representative of rainfall, tide and wind force and direction to recommend when and on which beach control samples should be taken. The validation of this software and methodological tool over the two summer periods of 2005 and 2006 enables to proceed towards a more complex operational system, which takes into account the continuous evolution of all the impacting factors.
\end{abstract}

\section{I INTRODUCTION}

Le contrôle et l'évaluation de l'impact des rejets urbains permettent une meilleure gestion d'un des volets importants de la qualité des eaux marines, à savoir l'aptitude sanitaire des eaux de baignade. Les systèmes d'assainissement collectifs sont constitués d'un système de collecte, les réseaux d'assainissement, et d'un système de traitement, les usines de dépollution des eaux usées, couramment appelées stations d'épurations. D'après le Ministère de la Santé [1], dans près de $40 \%$ des cas, les causes de pollution qui expliquent le classement de zones de baignade en catégorie C (pollution momentanée, 4,5\% des zones de baignade en 2005) ou en catégorie D (pollution très fréquente, $0,2 \%$ des zones de baignade en 2005) sont liées à l'assainissement :

- $15,5 \%$ pour insuffisance structurelle de l'assainissement par temps sec (absence de station d'épuration, traitement insuffisant, capacité du système insuffisante, mauvais branchements) ;

- $14,5 \%$ pour insuffisance structurelle de l'assainissement par temps de pluie, orage compris (mauvaise séparation eaux usées/eaux pluviales, rejets directs du réseau unitaire ou pluvial par temps de pluie) ;
- $8,2 \%$ pour dysfonctionnements ponctuels de l'assainissement (panne, rupture de canalisation, débordement du réseau par insuffisance d'entretien, dysfonctionnement de l'assainissement non collectif)

Ces insuffisances et ces dysfonctionnements ne sont pas l'apanage des zones de baignade classées en $\mathrm{C}$ ou en $\mathrm{D}$. Des actions sont mises en œuvre par les collectivités et les services de l'Etat, notamment l'amélioration de l'assainissement (construction d'ouvrages de stockage ou de traitement, travaux d'amélioration de ces derniers ou des réseaux, etc.), mais également l'information du public accompagnée le cas échéant de mesures de fermeture temporaire ou permanente de zones de baignade au cours de la saison balnéaire (Ministère de la Santé et des Solidarités, 2006).

Les dispositions de la nouvelle directive européenne, dénommée " gestion de la qualité des eaux de baignade » [2] vont inciter les collectivités locales à poursuivre les actions de prévention de la pollution et de maîtrise de ses causes, notamment à travers l'établissement des profils des eaux de baignade. Cette directive va également les amener à anticiper ces non-conformités, afin d'être en mesure de les gérer et de communiquer auprès du public. Enfin, en raison du durcissement des exigences sur la qualité microbiologique des eaux de baignade (Cf. Tableaux I et II), fortement influen- 
Tableau I. Qualité requise des eaux de baignade Extrait des exigences de la directive 76/160/CEE du 8 décembre 1975 [3].

\begin{tabular}{|l|c|c|}
\hline \multicolumn{1}{|c|}{ Paramètres } & $\begin{array}{c}\text { Valeur } \\
\text { Guide } \\
(/ \mathbf{1 0 0} \mathbf{~ m L})\end{array}$ & $\begin{array}{c}\text { Valeur } \\
\text { Impérative } \\
(/ / 100 ~ \mathbf{~ m})\end{array}$ \\
\hline Coliformes fécaux $(\approx$ E. coli $)$ & 100 & 2000 \\
\hline $\begin{array}{l}\text { Streptocoques fécaux } \\
(\approx \text { Entérocoques })\end{array}$ & 100 & - \\
\hline
\end{tabular}

Tableau II. Qualité requise des eaux de baignade Extrait des exigences de la directive 2006/7/CE du 15 février [2].

\begin{tabular}{|l|c|c|c|}
\hline Paramètres & $\begin{array}{c}\text { Excellente } \\
\text { qualité } \\
(/ \mathbf{1 0 0} \mathbf{~ m L})\end{array}$ & $\begin{array}{c}\text { Bonne } \\
\text { qualité } \\
(/ / 100 ~ \mathbf{~ m L})\end{array}$ & $\begin{array}{c}\text { Qualité } \\
\text { Suffisante } \\
(/ / 100 ~ \mathbf{~ m L})\end{array}$ \\
\hline E. coli & $250^{*}$ & $500^{*}$ & $500^{* *}$ \\
\hline Entérocoques & $100^{*}$ & $200^{*}$ & $185^{* *}$ \\
\hline
\end{tabular}

* Evaluation au $95^{\text {ème }}$ percentile, $* *$ Evaluation au $90^{\text {ème }}$ percentile.

cée par l'assainissement puisque les bactéries réglementées sont d'origines fécales, les efforts déjà entrepris devront être poursuivis voire renforcés, afin d'être à l'abri du classement demain d'une zone de baignade en qualité insuffisante, classée aujourd'hui en A (bonne qualité).

Dans ce contexte réglementaire en pleine évolution (les Etats Membres devront mettre en place les dispositions pour se conformer à cette nouvelle directive au plus tard le 24 mars 2008), il est intéressant pour les collectivités de se doter d'outils de maîtrise opérationnelle de la qualité des eaux de baignade de leur territoire.

\section{UNE EXPÉRIENCE PILOTE DE GESTION OPÉRATIONNELLE DE LA QUALITÉ DES EAUX DE BAIGNADE}

Le point de départ de la réflexion sur la qualité des eaux de baignade est l'établissement du profil de vulnérabilité de la plage. Celui-ci consiste en l'identification des flux de pollution auxquels elle est soumise et leur hiérarchisation au moyen de campagnes de mesure appropriées, par temps sec et par temps de pluie (Cf. Figure 1). De ce profil découle les moyens à mettre en ouvre pour juguler ces flux (travaux) et ceux pour maîtriser ou anticiper leurs impacts (gestion opérationnelle).

Afin de mettre en relation l'origine des flux de pollution bactériologique et l'impact sur une plage donnée, il convient de prendre en compte l'intervention de deux facteurs essentiels :

- la capacité de survie des micro-organismes dans l'environnement ;

- le transport et la dilution des bactéries provenant des effluents urbains par les courants.

Pour ce dernier, le recours à la modélisation hydrodynamique numérique est particulièrement utile en zone marine côtière. En effet, tandis que pour une baignade en rivière on peut établir un lien de causalité amont aval entre un rejet et

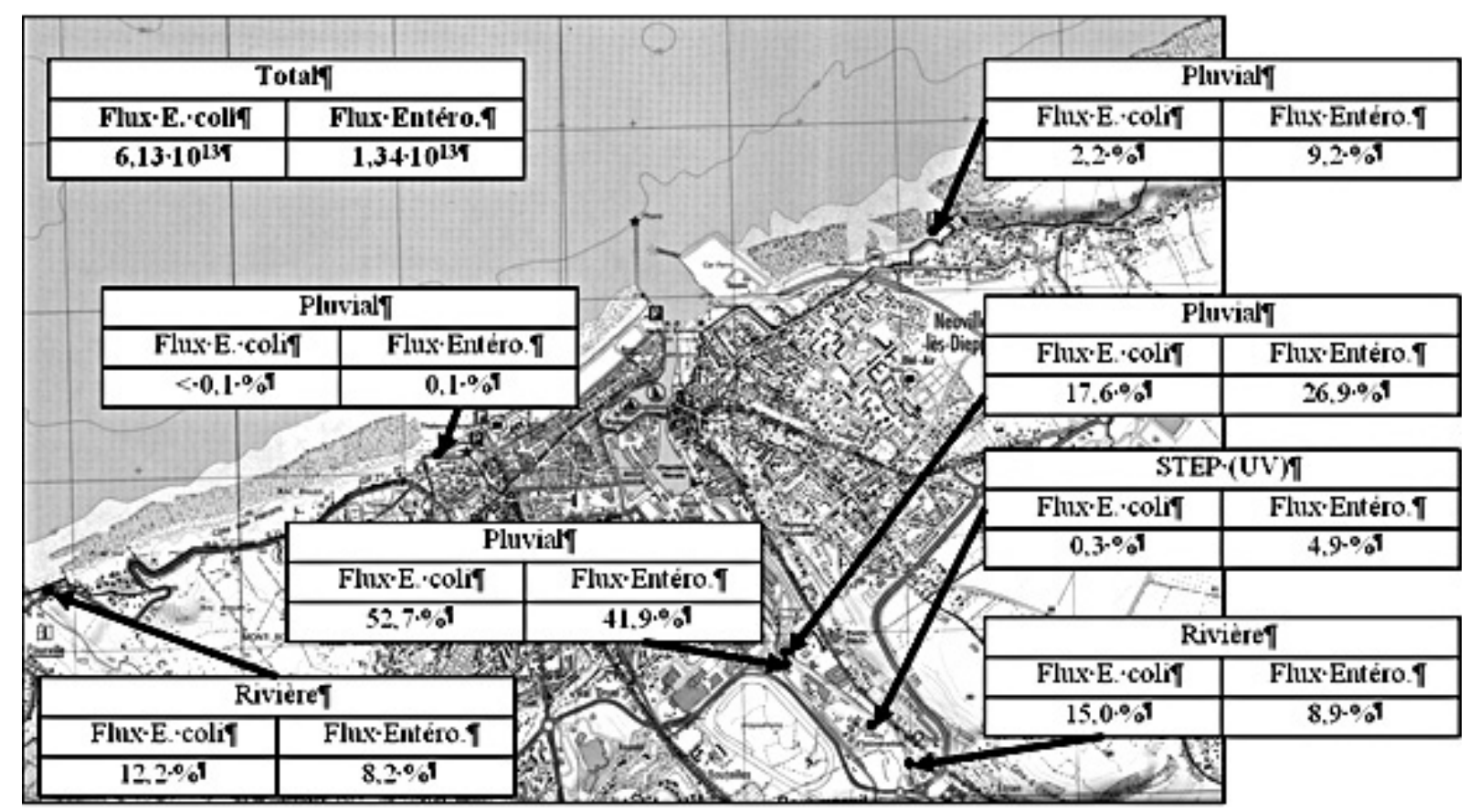

Figure 1 : Bilan des flux de pollution rejetés en mer au droit de Dieppe (Seine-Maritime) pendant la pluie du 29 août 2003 (10 $\mathrm{mm}$ en 2 heures). 
son impact sur une zone de baignade, en zone marine côtière ce lien de causalité est plus complexe à établir en raison de l'influence des courants (Cf. figures 2 a et $2 \mathbf{b}$ ). Un modèle hydrodynamique numérique tel MARS développé par l'Ifremer, peut prendre en compte les phénomènes suivants :
- l'amplitude de la marée ;

- les conditions atmosphériques (vent et pression);

- les débits des fleuves;

- la circulation océanique globale ;

— et l'état de la mer

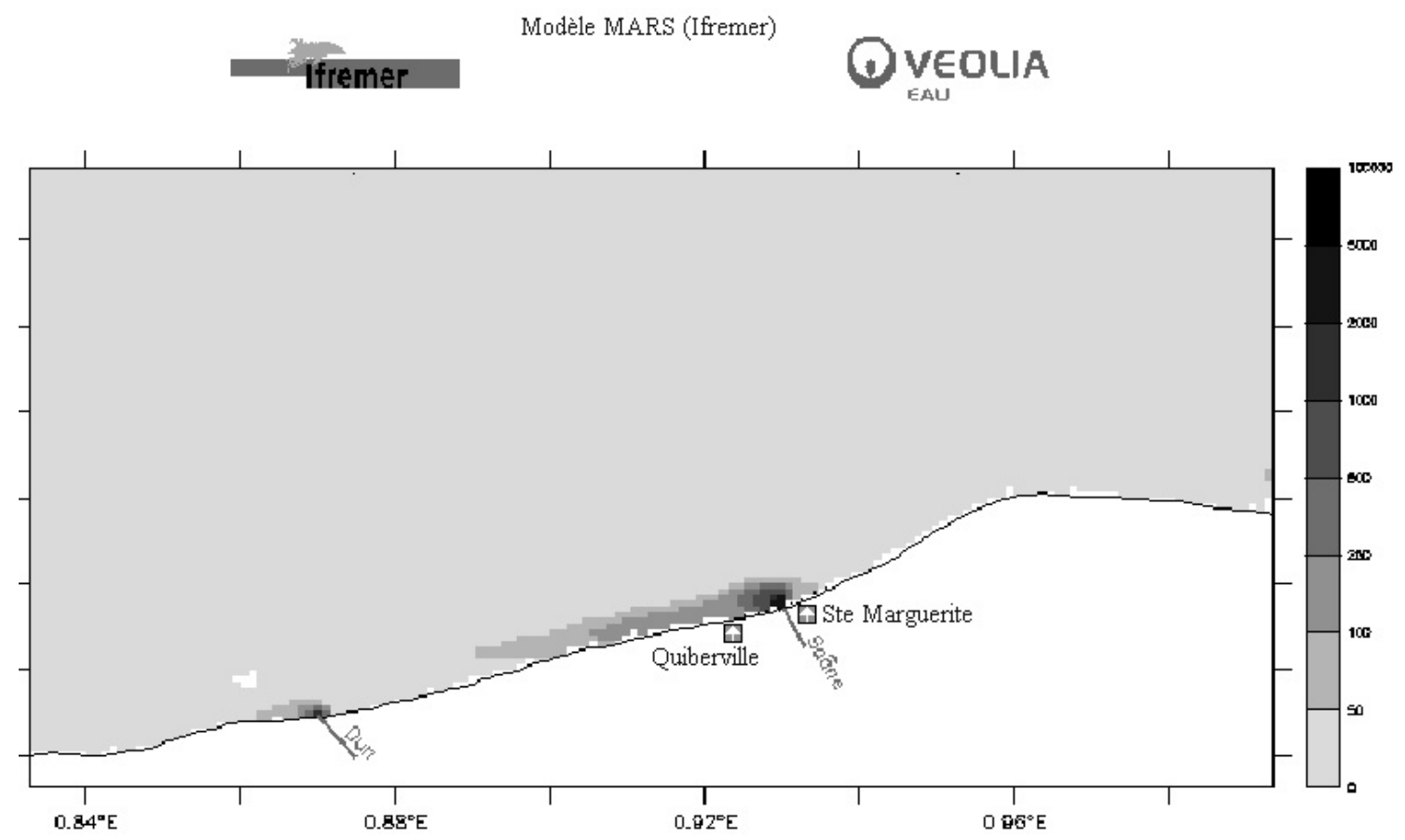

Figure 2a : Extension du panache d'E. coli provenant de la rivière Saâne à marée descendante (Pleine Mer $+4 \mathrm{~h} 45)$ : la plage de Quiberville est la plus touchée.



Figure 2b : Extension du panache d'E. coli provenant de la rivière Saâne à marée montante (Basse Mer $+1 \mathrm{h40})$ : la plage de Sainte-Marguerite est la plus touchée. 
A partir d'une bonne connaissance des flux de micro-organismes, leur localisation et leur intensité (par temps sec et par temps de pluie) et d'une étude de sensibilité du modèle, un outil de gestion opérationnelle a été bâti sur la base de scénarios pré-modélisés. Près d'une centaine de scénarios combinent des valeurs fixes des paramètres dont l'action est prépondérante sur l'intensité et l'extension des panaches :

- 2 coefficients de marée (morte eau moyenne et vive eau moyenne) ;

- direction et intensité de vent représentatives des régimes météorologiques les plus fréquents et les plus défavorables ; - des facteurs déterminant l'intensité des flux de microorganismes (pluie et antécédents pluvieux).

C'est ainsi que près d'une centaine de scénarios de référence ont été pris en compte par les équipes de Veolia et de l'Ifremer. Validée par une analyse de sensibilité et par un suivi rigoureux de la pertinence des prévisions introduites dans la base de données, cette méthode possède l'avantage de reposer sur des modélisations préalables.

Pendant la saison balnéaire, l'opérateur n'a besoin que de sélectionner les valeurs discrètes des paramètres se rapprochant le plus des cas pré-modélisés. Il est aidé en cela par une interface homme-machine (IHM) qui propose la saisie des informations pertinentes et sélectionne certains paramè- tres de façon automatique tels que le coefficient de marée en fonction de la date d'utilisation (Figure 3). Les résultats fournis instantanément par la consultation de la base de données de scénarios permettent d'évaluer les risques de dégradation de la qualité des eaux de baignade au cours des prochaines heures (Figure $4 \mathbf{a}$ et $\mathbf{4 b}$ ), avec comme consigne d'opérer des prélèvements complémentaires à un horaire clairement prescrit.

Ce système, dénommé SAERS (Système d'Aide à l'Evaluation des Risques Sanitaires), a été utilisé en routine par les équipes d'exploitation de Veolia Eau à Dieppe pendant les saisons estivales 2005 et 2006.

Il a pu être ainsi validé que l'usage d'un modèle courantologique côtier permet d'introduire une véritable dimension prédictive à la gestion opérationnelle, dans un contexte de courants de marée très marqués, comme c'est le cas sur le littoral du Pays de Caux.

\section{III $\square$ PERSPECTIVES : VERS UNE GESTION INTÉGRÉE DES REJETS D'ASSAINISSEMENT CÔTIERS}

Si la démarche précédemment décrite répond de façon pragmatique aux besoins opérationnels dans un environne-

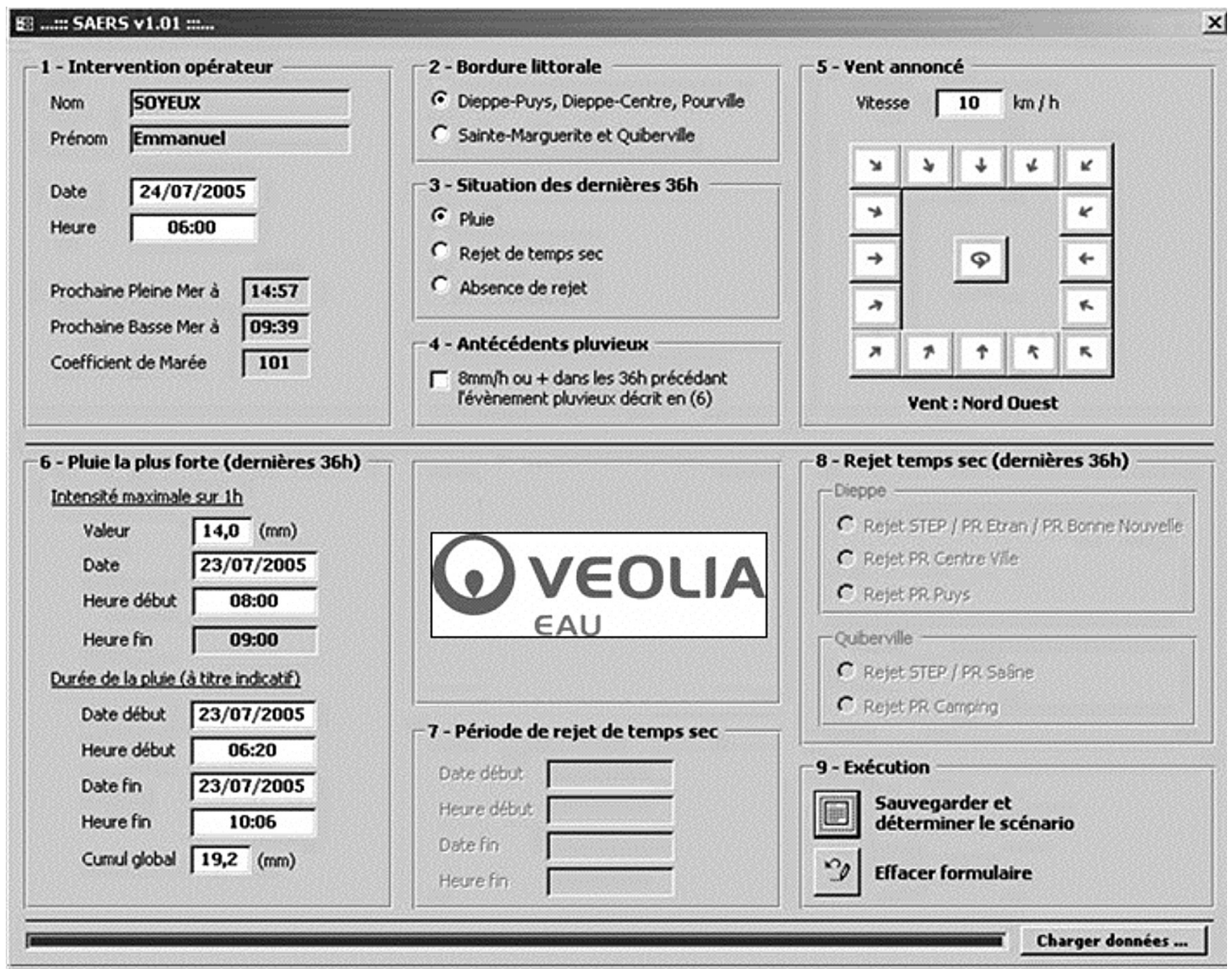

Figure 3 : Interface Homme-Machine utilisée par les opérateurs de Veolia Eau pour sélectionner chaque jour le scénario le plus proche de la situation réelle. 


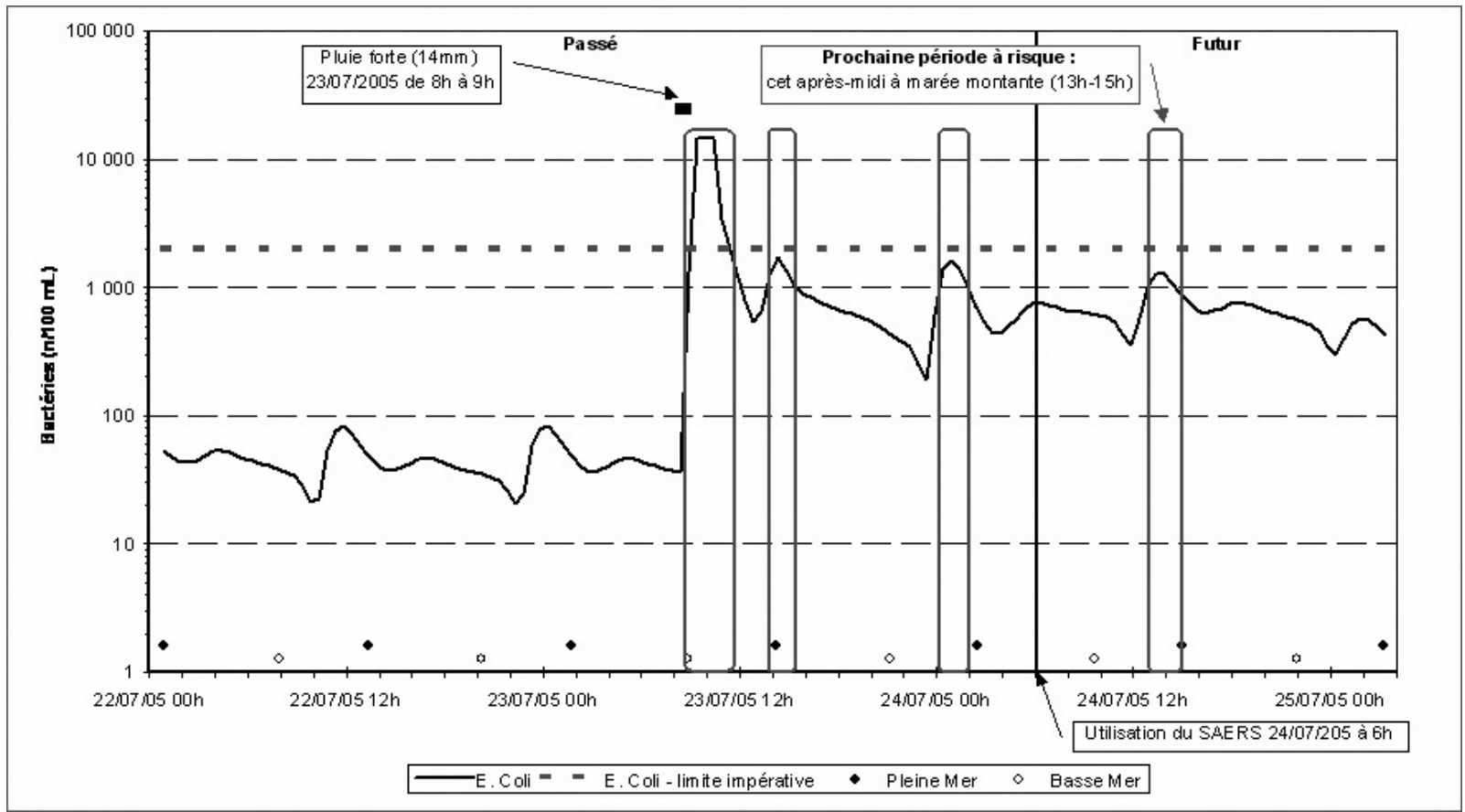

Figure 4a : Prévision de pollutogramme sur la plage de Puys. Les analyses rapides par la méthode ColiPlage ${ }^{\circledR}$ sont prescrits en fonction de l'heure à laquelle le pic se produit s'il est voisin du seuil réglementaire de $2000 \mathrm{E}$. coli/100 mL.
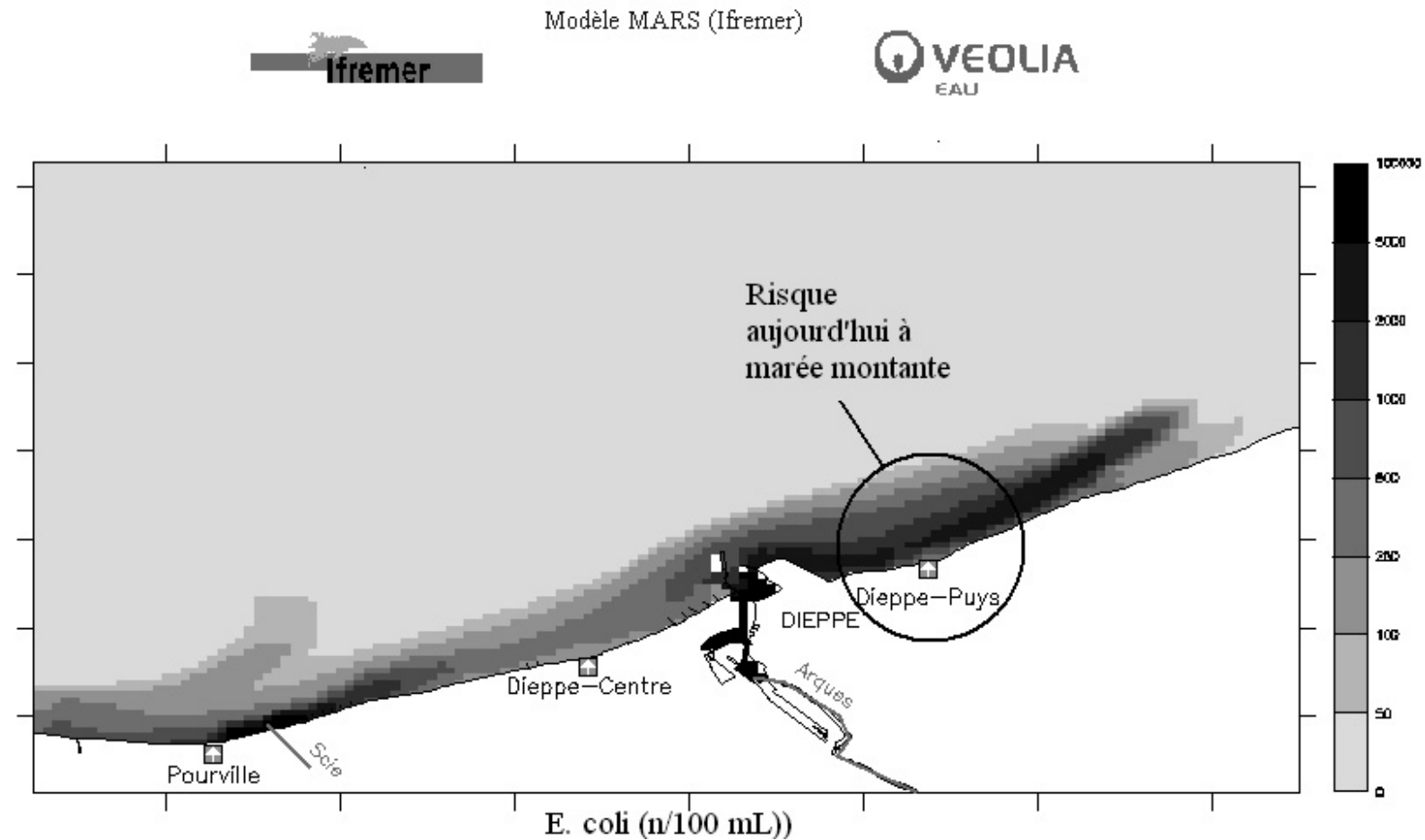

Figure 4b : Prévision de l'extension du panache d'E. coli sur 3 plages de l'agglomération de Dieppe associée à la situation de la figure $4 a$.

ment relativement simple, elle se heurte à une complexité combinatoire des scénarios s'il devient nécessaire d'affiner les prévisions. Tel est le cas dans une zone où les courants sont fortement réorientés par les effets météorologiques, ou bien lorsqu'il devient nécessaire de mieux appréhender la variabilité des débits et des flux des apports, ou encore en prenant en compte la dépendance effective de la survie bactérienne en milieu marin vis-à-vis de l'exposition à 
la lumière naturelle (ensoleillement), effet de la turbidité induite par la remise en suspension des sédiments sous l'effet des vagues, etc.

Pour ce faire, le recours à une modélisation validée devient systématique et quotidien. Elle seule permet de prendre en compte l'évolution temporelle continue de divers facteurs, tels que :

- une prévision météorologique détaillée et évolutive (ne plus se contenter de conditions types et constantes), une prise en compte quotidienne de la circulation océanique évoluant aux confins de la zone d'intérêt;

- une variation des débits et des flux des apports en introduisant les résultats de modèles de réseau d'assainissement ou d'usine de dépollution des eaux usées, ainsi que ceux des simulations des écoulements naturels des bassins versants ;

- une mémoire des phénomènes qui permet une réelle convolution entre la dynamique des apports et celle de la circulation littorale sur plusieurs jours ;

- une prévision d'état de mer et de son impact en termes de circulation et de brassage des sédiments fins ;

- une estimation précise des variations de la salinité et de la température dans les masses d'eau;

- une appréciation satisfaisante de la survie en mer des rejets bactériens, car leur mortalité, plus ou moins rapide, est modulée par l'ensemble des variations des paramètres précédents.
Le principe de la base de scénarios en nombre discret pourra donc être progressivement remplacé par une approche plus perfectionnée, mais la méthodologie de validation sera conservée car d'autant plus importante : l'ensemble des combinaisons de paramètres balayées par les conditions réelles mettront en effet les modèles à l'épreuve. Les équipes de recherche ont parfaitement conscience que la gageure demeure de concevoir un outil simple pour l'opérateur qui, au quotidien, interroge le système pour une prise de décision rapide et fiable. L'évolution de l'outil opérationnel repose donc sur la fourniture simultanée de résultats de modèle et de mesure : les progrès instrumentaux et ceux de la modélisation numérique se conjuguent par conséquent dans la prochaine génération des outils d'aide à la gestion des impacts des rejets d'assainissement sur les plages.

\section{REFERENCES ET CITATIONS}

[1] Ministère de la Santé et des Solidarités (2006). — Etat sanitaire des eaux de baignade en mer et en eau douce - bilan de la saison balnéaire 2005. 103 pp., Mai 2006.

[2] Directive 76/160/CEE du Conseil du 8 décembre 1975 concernant la qualité des eaux de baignade.

[3] Directive 2006/7/CE du Parlement Européen et du Conseil du 15 février 2006 concernant la gestion de la qualité des eaux de baignade. 